\title{
An Efficient Method for the Evaluation of Secular Effects in the Perturbed Keplerian Motion
}

\author{
László Á. Gergely, Zoltán Keresztes, Balázs Mikóczi \\ Departments of Theoretical and Experimental Physics, University of Szeged, Szeged 6720, \\ Hungary
}

\begin{abstract}
Binary systems subject to generic perturbations evolve on quasiperiodic orbits. We derive the most generic class of perturbations, which allow to evaluate secular effects via generalized complex true and eccentric anomaly parameters, by use of the residue theorem. Such perturbations include both the generic Brumberg force and various linear contributions to the conservative dynamics of compact binaries, up to the second post-Newtonian order. As an example, we compute the self-spin contribution to the luminosity due to gravitational radiation of a compact binary consisting of galactic black holes, the most important type of source for LISA. This translates to simply calculate the residue of the instantaneous energy loss in the origin of the complex parameter plane, which illustrates the power of the presented method.
\end{abstract}

Subject headings: compact binaries, perturbed Keplerian motion, parametrization

\section{Introduction}

The perturbed two-body problem is one of the central areas of interest of Celestial Mechanics. A wide class of perturbations of the dynamics of a binary system are described by the generic Brumberg force [Brumberg (1991)]. Another type of perturbation is provided by general relativistic modifications of gravitational dynamics. Such measurable effects arise in planetary motion (like the general relativistic contribution to the precession of the perihelion of the planet Mercury), however more important are the modifications in the strong-field regime, typically appearing in compact binaries composed of neutron stars and / or black holes. A well-known example is the Hulse-Taylor double pulsar [Hulse \& Taylor (1975)], the orbital period of which is continuously decreasing as gravitational radiation escapes from the system [Taylor, Fowler, McCulloch (1979)] (for a recent review see [Weisberg \& Taylor 
(2004)]). Efforts to directly detect gravitational waves with Earth-based interferometric detectors (LIGO[Abramovici et al. (1992)], VIRGO [Bradaschia et al. (1990)], GEO [Hough (1992)] and TAMA [Kuroda et al. (1997)]) are currently under way. Gravitational waves from distant coalescing galactic black hole binaries will be searched for by the forthcoming Laser Interferometer Space Antenna (LISA) [Bender et al. (1996)]. With improving detector sensitivity, upper limits for the signal emitted by different configurations of gravitational wave sources were already set ([Abbott et al. (2004)], [Messaritaki (2005)], [Abbott et al (2005)]). Capturing gravitational waves in the noisy background requires a 3.5 postNewtonian (PN) accuracy both in the orbital phase [Blanchet (2002)] and in the energy loss [Poisson (1995)] occurring in a compact binary.

The backreaction of the escaping gravitational radiation on the orbit decreases the orbital period of the compact binary. Although higher order corrections to this effect are too small to be directly detected in the early stages of the inspiral, they are important in the latter stages, when the post-Newtonian parameter increases up to 0.1 (at 10 gravitational radii separation) and higher order contributions become quite significant. Therefore the contributions to the energy loss $\langle d E / d t>$ and angular momentum loss $\langle d L / d t>$ have been worked out up to $2 \mathrm{PN}$ order accuracy in the wave generation (or equivalently, to 4.5 PN order following the Keplerian picture), in the case of eccentric orbits, and up to even higher orders for circular orbits. (Here $<>$ denotes the time-average over one period, e.g. a secular contribution.) The corrections arise at each PN order from both general relativistic modifications of the dynamics and/or as a manifestation of various physical characteristics of the system, like the spins, mass quadrupole momenta and magnetic dipole momenta of the constituents. The final aim of gravitational wave astronomy is to incorporate all of these physical characteristics into accurate templates to be used in data processing. Recovering the physical characteristics of astronomical objects from a simultaneous analysis of data collected in both the electromagnetic spectrum and gravitational wave bandwidth would be a major breakthrough in modern astronomy.

Driven by this motivation, the interest in the perturbed two-body problem has been renewed. In this context, in [Gergely, Perjés, Vasúth (2000)] a generic perturbed two-body problem with radial equation

$$
\begin{aligned}
\dot{r}^{2} & =\dot{r}_{N}^{2}+\sum_{i=0}^{p} \frac{\varphi_{i}}{\mu^{2} r^{i}} \\
\dot{r}_{N}^{2} & =2 \frac{E}{\mu}+2 \frac{G m}{r}-\frac{L^{2}}{\mu^{2} r^{2}}
\end{aligned}
$$

was considered. The point mass $\mu$ (representing the reduced mass of the binary) is orbiting a fixed gravitational centre $m$ (the total mass of the system) at distance $r$ (the separation of 
the components). The energy $E$ and the magnitude of the orbital angular momentum $L$ are constants of the perturbed motion ${ }^{1}$. The expression $\dot{r}_{N}^{2}$ (the subscript $N$ denoting Newtonian) has the familiary Newtonian functional form, however $E$ and $L$ characterize the perturbed motion. The perturbing terms contain the small coefficients $\varphi_{i}$, assumed constants. As will be shown in detail in Section 3, both the generic perturbing Brumberg force, the first postNewtonian order (1PN) general relativistic corrections [Damour \& Deruelle (1985)] and the spin-orbit (SO) interaction for compact binaries [Barker \& O'Connell (1979)] fit into this scheme. The generic equation (1) defines a radial period $T$ and turning points $r_{\min }$.

With these assumptions the integrals of the type

$$
I(\omega, n)=\int_{0}^{T} \frac{\omega}{r^{2+n}} d t
$$

(with constant $\omega$ and $n$ arbitrary integer) could be easily evaluated by use of the residue theorem. Important steps in the proof of [Gergely, Perjés, Vasúth (2000)] were the introduction of suitable generalized true and eccentric anomaly parametrizations of the radial motion, $r(\chi)$ and $r(\xi)$ as

$$
\begin{aligned}
\frac{2}{r} & =\frac{1+\cos \chi}{r_{\min }}+\frac{1-\cos \chi}{r_{\max }}, \\
2 r & =(1+\cos \xi) r_{\min }+(1-\cos \xi) r_{\max }
\end{aligned}
$$

together with their complex counterpart $z=\exp (i \chi)$ and $w=\exp (i \xi)$, respectively. The advantage of this method over other methods for computing the secular integrals (3) is its striking simplicity. As proven in [Gergely, Perjés, Vasúth (2000)], in the majority of physically relevant cases the only pole is in the origin. Extremely rarely, a second pole (also given) occurs. The method was successfully applied in [Gergely, Perjés, Vasúth (1998)] for the evaluation of the averaged energy and angular momentum losses due to the SO interaction for compact binaries on elliptic orbit, and the results were in agreement with those of [Rieth \& Schäfer (1997)], obtained by a different method.

The more accurate study of the inspiral phase of compact binaries however reveals other important contributions to be taken into account. These are the perturbations due to the (a) spin-spin (SS) interaction [Barker \& O'Connell (1979)]; (b) the interaction of the magnetic dipole moments for neutron stars with huge magnetic fields (DD) [Ioka \& Taniguchi (2000)] and (c) the contribution arising from the motion of one of the binary components (treated as a monopole) in the quadrupolar field of the other (QM) [Poisson (1998)]. The

\footnotetext{
${ }^{1}$ Separating $E$ and $L$ from the rest of the coefficients of $r^{0}$ and $r^{-2}$ in the radial equation is advantageous, as it will enable us to express all results in terms of constants with physical interpretation.
} 
corresponding secular contributions to the energy and angular momentum losses could be computed [Gergely (2000)], [Vasúth, Keresztes, Mihály, Gergely (2003)], and [Gergely \& Keresztes (2003)] by the method presented in [Gergely, Perjés, Vasúth (2000)], in spite of the radial equation being different from Eq. (1), which rendered these computations outside the domain of validity of Theorems 1 and 2 of [Gergely, Perjés, Vasúth (2000)]. Therefore the question arose, whether the results presented in [Gergely, Perjés, Vasúth (2000)] would hold more generically.

In all above mentioned cases the radial equation was a generalization of Eq. (1) with the coefficients $\varphi_{i}$ being periodic functions of the true anomaly $\chi$. Thus in this paper we raise the question, whether the results of [Gergely, Perjés, Vasúth (2000)] would hold for generic periodic functions $\varphi_{i}(\chi)$. The answer we find is that some restrictions apply, and we derive the class of admissible periodic functions $\varphi_{i}$. Section 2 contains the announcement and proof of the generalized Theorems 1 and 2, as compared to [Gergely, Perjés, Vasúth (2000)].

Starting from the explicit expressions for the respective interactions, we show in Section 3, that the SS, QM and DD perturbations fit into the class of admissible perturbing functions $\varphi_{i}$.

In Section 4, as a simple application of the presented method, we give the self-interaction spin contributions to the luminosity of a compact binary consisting of black holes, in the case when $S_{2} \ll S_{1}$.

\section{Admissible perturbations and the integration method}

The turning points of the radial motion (1), with $\varphi_{i}=\varphi_{i}(\chi)$ are the loci $\dot{r}=0$, given by:

$$
r_{\min }=\frac{G m \mu \pm A_{0}}{-2 E} \pm \frac{1}{2 \mu A_{0}} \sum_{i=0}^{p} \varphi_{i}^{ \pm}\left[\frac{\mu\left(G m \mu \mp A_{0}\right)}{L^{2}}\right]^{i-2} .
$$

Here $A_{0}$ represents the magnitude of the Laplace-Runge-Lenz vector characterizing a Keplerian motion with $E$ and $L$ and we have introduced the notations

$$
\varphi_{i}^{-}=\varphi_{i}(0), \quad \varphi_{i}^{+}=\varphi_{i}(\pi)
$$

Unless in the case presented in [Gergely, Perjés, Vasúth (2000)], here $\varphi_{i}^{-} \neq \varphi_{i}^{+}$. The expression of the turning points allow to write up the parametrizations (4) and (5) in detail, whenever needed. 
The integrals (3) could be formally rewritten in terms of the integration variable $\chi$ as

$$
I(\omega, n)=\int_{0}^{2 \pi} \frac{\omega}{r^{n}}\left(\frac{1}{r^{2}} \frac{d t}{d \chi}\right) d \chi .
$$

\subsection{The case $n \geq 0$}

For $n \geq 0$ the factor $r^{n}$ in the denominator of the integrand in Eq. (8) has the binomial expansion

$$
\left[\frac{2}{r(\chi)}\right]^{n}=\sum_{k=0}^{n}\left(\begin{array}{l}
n \\
k
\end{array}\right)\left(\frac{1+\cos \chi}{r_{\min }}\right)^{k}\left(\frac{1-\cos \chi}{r_{\max }}\right)^{n-k} .
$$

By applying the chain rule $d t / d \chi=\dot{r}^{-1} d r / d \chi$, with $d r / d \chi$ derived from the true anomaly parametrization (4), and inserting $\dot{r}^{-1}$ as the series expansion of the radial equation (1), we find for the second factor of the integrand (8)

$$
\begin{aligned}
\frac{1}{r^{2}} \frac{d t}{d \chi}= & \frac{\mu}{L}\left(1+\frac{L^{2}}{4 \mu^{2} A_{0}^{2} \sin ^{2} \chi} \Gamma\right) \\
\Gamma= & \sum_{i=0}^{p}\left(\Delta_{+}^{i}+\Delta_{-}^{i} \cos \chi-\frac{2 \varphi_{i}(\chi)}{r^{i}}\right) \\
\Delta_{ \pm}^{i}= & \frac{\mu^{i}}{L^{2 i}}\left[\varphi_{i}^{-}\left(G m \mu+A_{0}\right)^{i}\right. \\
& \left. \pm \varphi_{i}^{+}\left(G m \mu-A_{0}\right)^{i}\right]
\end{aligned}
$$

The second term of the expression (10) contains $\sin ^{2} \chi$ in the denominator, and apparently becomes singular at $\chi=k \pi$ ( $k$ any integer). The key point of the proof of Theorem 1 of [Gergely, Perjés, Vasúth (2000)] was that a factor of $\sin ^{2} \chi$ could be also separated from $\Gamma$, so that the integrand of $I(\omega, n)$ became regular everywhere. The proof has also showed that there is no other $\chi$-dependence left in the denominator.

We would like to see whether this convenient property of $\Gamma$ holds for generic periodic functions $\varphi_{i}(\chi)$. For this we decompose $\varphi_{i}(\chi)$ as follows

$$
\varphi_{i}(\chi)=\sum_{j=0}^{\infty}\left(f_{i j}+g_{i j} \cos \chi\right) \sin ^{j} \chi,
$$

the coefficients $f_{i j}$ and $g_{i j}$ being constants. Then the condition

$$
\varphi_{i}^{\mp}=f_{i 0} \pm g_{i 0}
$$


follows. Eq. (13) is equivalent with a decomposition into a generic Fourier series, however better suited for our purposes. Its advantage lies in the property of the terms with $j \geq 2$, of manifestly containing the factor $\sin ^{2} \chi$. Therefore singular integrands in (8) could arise only from the terms containing $f_{i 0}, g_{i 0}, f_{i 1}$ and $g_{i 1}$. We will discuss these one by one.

As $f_{i 0}$ is the constant part of $\varphi_{i}(\chi)$, the contributions containing $f_{i 0}$ are the constant perturbing functions already dealt with in [Gergely, Perjés, Vasúth (2000)]. Thus the terms containing $f_{i 0}$ are regular.

A similar algebra as for the terms with $f_{i 0}$ applies to the terms containing $g_{i 0}$. By employing the binomial expansion (9) and the expression of the turning points (6), those contributions to $\Gamma$ which contain the factor $2\left(\mu / L^{2}\right)^{i} g_{i 0}$ sum up to

$$
\sum_{l=0}^{i}\left(\begin{array}{l}
i \\
l
\end{array}\right)(G m \mu)^{i-l} A_{0}^{l}\left[\frac{1-(-1)^{l}}{2} \sin ^{2} \chi+\Xi_{l+1}(\chi)\right]
$$

The expressions $\Xi_{k}(\chi)$ are defined by Eqs. (29)-(31) of [Gergely, Perjés, Vasúth (2000)], and they are proportional to $\sin ^{2} \chi$. This proves that the terms with $g_{i 0}$ do not give singular contributions to the integrand of $I(\omega, n)$.

What remains to check are the $j=1$ terms of $\Gamma$, given by

$$
-\sum_{i=0}^{p} \frac{2\left(f_{i 1}+g_{i 1} \cos \chi\right) \sin \chi}{r^{i}}
$$

(We have used that, according to Eq. (14), the expressions $\Delta_{ \pm}^{i}$ receive no contribution from $f_{i 1}$ and $g_{i 1}$.) As the coefficients $f_{i 1}, g_{i 1}$ are already of first order, the Keplerian true anomaly parametrization $r=\left(\mu / L^{2}\right)\left(G m \mu+A_{0} \cos \chi\right)$ can be employed in Eq. (16). A tedious but straightforward algebra, based on (a) the binomial expansion of $1 / r^{i}$; (b) the replacement $\cos ^{2} \chi=1-\sin ^{2} \chi$, whenever possible; (c) a second binomial expansion of $\left(1-\sin ^{2} \chi\right)^{q}$,

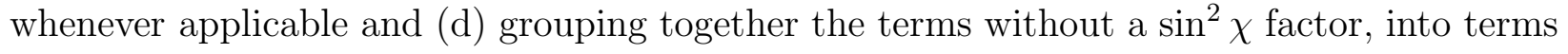
proportional to $\sin \chi$ and $\sin \chi \cos \chi$, leads to the following two conditions on the coefficients $f_{i 1}$ and $g_{i 1}$ :

$$
\sum_{i=0}^{p} \frac{\mu^{i}}{L^{2 i}}\left(G m \mu \pm A_{0}\right)^{i}\left(f_{i 1} \pm g_{i 1}\right)=0,
$$

which have to be satisfied in order $d t / d \chi$ to be regular for every $\chi$.

The last question to be addressed is what are the conditions to be imposed on $\omega$, in order that $I(\omega, n)$ stays regular. The answer is simple: $\omega$ can be any periodic function of $\chi$, which is regular. Such functions will not affect the proof presented above. 
With these conditions satisfied, all factors in the integrand of $I(\omega, n)$ become simple polynomials in $\sin \chi=\left(z^{2}-1\right) / 2 i z$ and $\cos \chi=\left(z^{2}+1\right) / 2 z$, provided $n \geq 0$. This renders the only pole in the origin, and we have proven:

Theorem 1: For all perturbed Keplerian motions characterized by the radial equation (1), with periodic perturbing functions $\varphi_{i}$ obeying the conditions (13) and (17), and for arbitrary periodic functions $\omega$ the integral $I(\omega, n \geq 0)$ is given by the residue in the origin of the complex true-anomaly parameter plane.

\subsection{The case $n<0$}

In order to cover the complementary case of $n<0$, we employ the eccentric anomaly parametrization (5). The integrals $I(\omega, n)$ then are evaluated as

$$
I(\omega, n)=\int_{0}^{2 \pi} \frac{\omega}{r^{n+1}}\left(\frac{1}{r} \frac{d t}{d \xi}\right) d \xi
$$

and for any $n^{\prime} \equiv-n-1 \geq 0$ we apply the binomial expansion

$$
(2 r)^{n^{\prime}}=\sum_{k=0}^{n^{\prime}}\left(\begin{array}{l}
k \\
n^{\prime}
\end{array}\right) r_{\min }^{k} r_{\max }^{n^{\prime}-k}(1+\cos \xi)^{k}(1-\cos \xi)^{n^{\prime}-k}
$$

which is a polynomial in $\cos \xi$. From the eccentric anomaly parametrization (5) and the radial equation $(1)$ (with $\varphi(\chi(\xi))$ in place of $\varphi(\chi))$ we find

$$
\begin{aligned}
& \frac{1}{r} \frac{d t}{d \xi}=\sqrt{\frac{\mu}{-2 E}}\left(1-\frac{E}{2 \mu A_{0}^{2} \sin ^{2} \xi} \Upsilon\right) \\
& \Upsilon=\sum_{i=0}^{p}\left(\Omega_{+}^{i}-\Omega_{-}^{i} \cos \xi-\frac{2 \varphi_{i}(\xi)}{r^{i-2}}\right) \\
& \Omega_{ \pm}^{i}=\left(\frac{\mu}{L^{2}}\right)^{i-2}\left[\varphi_{i}^{+}\left(G m \mu-A_{0}\right)^{i-2}\right. \\
&\left. \pm \varphi_{i}^{-}\left(G m \mu+A_{0}\right)^{i-2}\right]
\end{aligned}
$$

Here $\varphi_{i}^{-}$and $\varphi_{i}^{+}$are identical with those defined in terms of the true anomaly (this is, because at $r_{\max }$ both angles $\chi$ and $\xi$ take the values 0 and $\pi$, respectively). Considerations completely analogous to those in the proof of Theorem 1, together with the relation between the two parametrizations

$$
\begin{aligned}
\cos \chi & =\frac{G m \mu \cos \xi-A_{0}}{G m \mu-A_{0} \cos \xi}, \\
\sin \chi & =\frac{\left(-2 E L^{2} / \mu\right)^{1 / 2} \sin \xi}{G m \mu-A_{0} \cos \xi},
\end{aligned}
$$


after lengthy computations lead to the following result. Provided the Eqs. (17) hold, a factor of $\sin ^{2} \xi$ separates out from $\Upsilon$ and cancels out the $\sin ^{2} \xi$ factor of the denominator in Eq. (20).

As main difference with respect to the true anomaly parametrization, the terms $\varphi_{i}(\xi) / r^{i-2}$ in the expression (21) could lead to additional factors $\left(G m \mu-A_{0} \cos \xi\right)$ in the denominator of the integrand. Therefore, the second pole

$$
w_{1}=\left(\frac{G m \mu^{2}-\sqrt{-2 \mu E L^{2}}}{G m \mu^{2}+\sqrt{-2 \mu E L^{2}}}\right)^{1 / 2}
$$

could appear in the process of passing to a complex eccentric anomaly parameter. We can now enounce our

Theorem 2: For all perturbed Keplerian motions characterized by the radial equation (1), with periodic perturbing functions $\varphi_{i}$ obeying the conditions (13) and (17), and for arbitrary periodic functions $\omega$, the integral $I(\omega, n<0)$ is given by the sum of the residues in the origin of the complex eccentric-anomaly parameter plane and (for any $f_{i j}$ with $i+j>2$ or $g_{i j}$ with $i+j>1$ in $\varphi_{i}$ ) at $w_{1}$.

Our Theorems contain as special cases the Theorems presented in [Gergely, Perjés, Vasúth (2000)]. There, the conditions (17) are automatically satisfied, as $\varphi_{i}$ are constants. It is remarkable, that the constancy of $\varphi_{i}$ can be relaxed in a quite generic way, in which the infinite number of coefficients in the expansion of $\varphi_{i}(\chi)$ should obey only the two contraints (17).

\section{Admissible dynamical systems}

In this Section we present two important classes of dynamical systems, for which our Theorems apply.

\subsection{Perturbations characterized by the generic Brumberg force}

The generic perturbing Brumberg force [Brumberg (1991)], [Soffel (1988)], includes a wide range of perturbations employed in Celestial Mechanics. It can be derived from the Lagrangian

$$
\mathcal{L}_{B}=\mathcal{L}_{N}+\mathcal{L}_{P B}
$$




$$
\begin{aligned}
\mathcal{L}_{N}= & \frac{\mu \mathbf{v}^{2}}{2}+\frac{G m \mu}{r}, \\
\mathcal{L}_{P B}= & \frac{1}{4 c^{2}}\left(\alpha-\beta+\frac{\lambda}{2}\right) \mu \mathbf{v}^{4}+\alpha \frac{G m \mu}{c^{2} r^{3}}(\mathbf{r v})^{2} \\
& +\left(\beta-\alpha+\frac{\lambda}{2}\right) \frac{G m \mu}{c^{2} r} \mathbf{v}^{2}+\left(\beta-\gamma+\frac{\lambda}{2}\right) \frac{G^{2} m^{2} \mu}{c^{2} r^{2}} .
\end{aligned}
$$

where $\alpha, \beta, \gamma$ and $\lambda$ are perturbation parameters. The radial equation derived in [Gergely, Perjés, Vasúth (2000)] from this Lagrangian is of the form (1), with the small coefficients

$$
\begin{aligned}
c^{2} \varphi_{0}^{B}= & -3(2 \alpha-2 \beta+\lambda) E^{2}, \\
c^{2} \varphi_{1}^{B}= & -4 G m \mu(3 \alpha-2 \beta+2 \lambda) E, \\
c^{2} \varphi_{2}^{B}= & -2 \mu^{2} G^{2} m^{2}(3 \alpha-2 \beta+2 \lambda+\gamma) \\
& +\frac{2}{\mu}(2 \alpha-2 \beta+\lambda) E L^{2} \\
c^{2} \varphi_{3}^{B}= & 2 G m(\alpha+2 \lambda) L^{2} .
\end{aligned}
$$

As all $\varphi_{i}^{B}$ are constants, these perturbations fit into the domain of validiy of the methods discussed in [Gergely, Perjés, Vasúth (2000)].

\subsection{Orbital evolution of compact binaries}

Another important example is provided by compact binary systems, consisting of black holes / neutron stars. Their dynamics is conservative up to the $2 \mathrm{PN}$ order, the first dissipative effects due to gravitational radiation occuring at 2.5 PN order. The conservative dynamics including all leading order contributions, due to either various physical characteristics, or to the general relativistic modifications of the motion is described by the Lagrangian

$$
\mathcal{L}_{C B}=\mathcal{L}_{N}+\mathcal{L}_{P N}+\mathcal{L}_{S O}+\mathcal{L}_{S S}+\mathcal{L}_{Q M}+\mathcal{L}_{D D}
$$

with the various contributions derived first in [Damour \& Deruelle (1985)] (PN), [Keresztes, Mikóczi, Gergely (2005)], (SO), [Kidder, Will, Wiseman (1993)] (SS), [Poisson (1998)] (QM) and [Ioka \& Taniguchi (2000)] (DD) $\operatorname{read}^{2}$ :

$$
\mathcal{L}_{P N}=\frac{1}{8 c^{2}}(1-3 \eta) \mu v^{4}+\frac{G m \mu}{2 r c^{2}}\left[(3+\eta) v^{2}+\eta \dot{r}^{2}-\frac{G m}{r}\right],
$$

\footnotetext{
${ }^{2}$ The magnitude and direction of the spins are denoted as $S_{i}$ and $\hat{\mathbf{S}}_{\mathbf{i}}$. The angle subtended by them is $\gamma=\cos ^{-1}\left(\hat{\mathbf{S}}_{\mathbf{1}} \cdot \hat{\mathbf{S}}_{\mathbf{2}}\right)$. The total spin is $\mathbf{S}=\mathbf{S}_{\mathbf{1}}+\mathbf{S}_{\mathbf{2}}$ and $\boldsymbol{\sigma}=\left(m_{2} / m_{1}\right) \mathbf{S}_{\mathbf{1}}+\left(m_{1} / m_{2}\right) \mathbf{S}_{\mathbf{2}}$. The magnitude and direction of the magnetic dipole moments $\mathbf{d}_{\mathbf{i}}$ are denoted as $d_{i}$ and $\hat{\mathbf{d}}_{\mathbf{i}}$. They subtend the angle $\lambda=\cos ^{-1}\left(\hat{\mathbf{d}}_{\mathbf{1}} \cdot \hat{\mathbf{d}}_{\mathbf{2}}\right)$ with each other. In a coordinate systems $\mathcal{K}$ with the axes $(\hat{\mathbf{c}}, \hat{\mathbf{L}} \times \hat{\mathbf{c}}, \hat{\mathbf{L}})$, where
} 


$$
\begin{aligned}
\mathcal{L}_{S O} & =\frac{G \mu}{2 c^{2} r^{3}} \mathbf{v} \cdot[\mathbf{r} \times(4 \mathbf{S}+3 \boldsymbol{\sigma})] \\
\mathcal{L}_{S S} & =\frac{G}{c^{2} r^{3}}\left[\left(\mathbf{S}_{\mathbf{1}} \cdot \mathbf{S}_{\mathbf{2}}\right)-\frac{3}{r^{2}}\left(\mathbf{r} \cdot \mathbf{S}_{\mathbf{1}}\right)\left(\mathbf{r} \cdot \mathbf{S}_{\mathbf{2}}\right)\right] \\
\mathcal{L}_{Q M} & =\frac{G \mu m^{3}}{2 r^{5}} \sum_{i=1}^{2} p_{i}\left[3\left(\hat{\mathbf{S}}_{\mathbf{i}} \cdot \mathbf{r}\right)^{2}-r^{2}\right] \\
\mathcal{L}_{D D} & =\frac{1}{r^{3}}\left[3\left(\mathbf{n} \cdot \mathbf{d}_{\mathbf{1}}\right)\left(\mathbf{n} \cdot \mathbf{d}_{\mathbf{2}}\right)-\mathbf{d}_{\mathbf{1}} \cdot \mathbf{d}_{\mathbf{2}}\right]
\end{aligned}
$$

Note that the $1 \mathrm{PN}$ accurate relativistic corrections to the Keplerian motion characterized by $\mathcal{L}_{P N}$ is the particular case of the perturbing Brumberg Lagrangian $\mathcal{L}_{P B}$, with the specifications $\alpha=\eta / 2, \beta=(1+3 \eta) / 2, \gamma=2+\eta, \lambda=2-\eta$ (where $\eta=\mu / m)$. Therefore the PN perturbations fit into the domain of validiy of the methods discussed in [Gergely, Perjés, Vasúth (2000)].

The same holds for the SO perturbations, where the only non-vanishing $\varphi_{i}^{S O}$ in the radial equation is

$$
\varphi_{3}^{S O}=-\frac{G \mu}{c^{2}}(4 \mathbf{L} \cdot \mathbf{S}+3 \mathbf{L} \cdot \boldsymbol{\sigma})
$$

This is a constant, as the angles $\kappa_{i}$ span by the spins with the orbital angular momentum $\mathbf{L}$ are constants (to leading order) ${ }^{3}$.

The situation is entirely different for the SS, QM and DD contributions to the dynamics of a compact binary. In all these cases the energy $E$ is a constant of motion, while (in contrast with the 1PN and SO contributions) the magnitude $L$ of the orbital angular momentum is not. Indeed, with the notable exception of the PN contribution, it can be derived from the respective Lagrangians that the orbital angular momentum $\mathbf{L} \equiv \mathbf{r} \times \mathbf{p}$ is not conserved: $\dot{\mathbf{L}} \neq$

$\hat{\mathbf{c}}$ is the unit vector in the $\mathbf{J} \times \mathbf{L}$ direction, the polar angles $\kappa_{i}$ and $\psi_{i}$ of the spins are defined as $\hat{\mathbf{S}}_{\mathbf{i}}=\left(\sin \kappa_{i} \cos \psi_{i}, \sin \kappa_{i} \sin \psi_{i}, \cos \kappa_{i}\right)$ (see [Gergely, Perjés, Vasúth (1998)]). In the coordinate system $\mathcal{K}^{i}$ with the axes $\left(\hat{\mathbf{b}}_{\mathbf{i}}, \hat{\mathbf{S}}_{\mathbf{i}} \times \hat{\mathbf{b}}_{\mathbf{i}}, \hat{\mathbf{S}}_{\mathbf{i}}\right)$, where $\hat{\mathbf{b}}_{\mathbf{i}}$ are the unit vectors in the $\mathbf{S}_{\mathbf{i}} \times \mathbf{L}$ directions, respectively, the polar angles $\alpha_{i}$ and $\beta_{i}$ of the the magnetic dipole moments $\mathbf{d}_{\mathbf{i}}$ are $\hat{\mathbf{d}}_{\mathbf{i}}=\left(\sin \alpha_{i} \cos \beta_{i}, \sin \alpha_{i} \sin \beta_{i}, \cos \alpha_{i}\right)(\operatorname{see}[$ Vasúth, Keresztes, Mihály, Gergely (2003)]). The quadrupolar parameters (see [Gergely \& Keresztes (2003)]) are defined as $p_{i}=Q_{i} / m_{i} m^{2}$, where $Q_{i}$ is the quadrupole-moment scalar [Poisson (1998)] of the $i^{\text {th }}$ axially symmetric binary component with symmetry axis $\hat{\mathbf{S}}_{\mathbf{i}}$.

${ }^{3}$ Note however that neither $\mathcal{L}_{S O}$ nor the radial equation with the perturbing function (32) characterize uniquely the SO-perturbation. This is, because a spin-supplementary condition (SSC) should be additionally imposed. We have followed here the SSC of [Pryce (1948)] and [Newton \& Wigner (1949)]. There are other known SSC-s, including the covariant SSC employed in [Kidder (1995)], which gives the non-vanishing constant perturbations $\varphi_{2}^{S O, \operatorname{cov}}=\left(2 E / c^{2} m\right)(\mathbf{L} \cdot \boldsymbol{\sigma})$ and $\varphi_{3}^{S O, \operatorname{cov}}=-\left(2 G \mu / c^{2}\right)(2 \mathbf{L} \cdot \mathbf{S}+\mathbf{L} \cdot \boldsymbol{\sigma})$. These can again be expressed in terms of the angles $\kappa_{i}$, which are constants in the leading order, required here. 
0 . While for SO perturbations this means merely a precessional change about the vector $4 \mathrm{~S}+3 \cdot \boldsymbol{\sigma}$ (so that $L$ is conserved), the situation is more complicated in the SS, QM and DD cases, in which $L$ is a function of the orbital position $L=L(\chi)$. In all these cases however an angular average $\bar{L}$ over the radial motion can be computed, which is conserved to the accuracy of linear effects we consider here. The reason for this is that up to $2 \mathrm{PN}$, the dynamics is conservative. A description in terms of the constants of motion $E$ and $\bar{L}$ is then in order, as described in detail in ([Gergely (2000) ], [Gergely \& Keresztes (2003)] and [Vasúth, Keresztes, Mihály, Gergely (2003)]. The radial equation in all these cases can be expressed in the form (1), with $\bar{L}$ in place of $L$ while the perturbing terms contain the following explicit $\chi$-dependences:

$$
\begin{aligned}
\varphi_{2}^{S S}= & \frac{G \mu^{2}}{2 c^{2} \bar{L}^{3}} S_{1} S_{2} \sin \kappa_{1} \sin \kappa_{2}\{2 \bar{A} \cos (\chi+\delta)] \\
& +(3 G m \mu+2 \bar{A} \cos \chi) \cos (2 \chi+\delta)\} \\
\varphi_{3}^{S S}= & \frac{G \mu}{c^{2}} S_{1} S_{2}\left[3 \cos \kappa_{1} \cos \kappa_{2}-\cos \gamma\right. \\
& \left.-3 \sin \kappa_{1} \sin \kappa_{2} \cos (2 \chi+\delta)\right] \\
\varphi_{2}^{Q M}= & -\frac{G m^{3} \mu^{3}}{2 \bar{L}^{2}} \sum_{i=1}^{2} p_{i} \sin ^{2} \kappa_{i}\left\{2 \bar{A} \cos \left(\chi+\delta_{i}\right)\right. \\
& \left.+(3 G \mu m+2 \bar{A} \cos \chi) \cos \left(2 \chi+\delta_{i}\right)\right\} \\
\varphi_{3}^{Q M}= & -G \mu^{2} m^{3} \sum_{i=1}^{2} p_{i}\left[1-3 \sin { }^{2} \kappa_{i} \cos { }^{2}\left(\chi+\frac{\delta_{i}}{2}\right)\right] \\
\varphi_{2}^{D D}= & -\frac{\mu^{2} d_{1} d_{2}}{\bar{L}^{2}}\left[(3 G m \mu+4 \bar{A} \cos \chi) \mathcal{B}_{2}(\chi)-\bar{A} \sin \chi \mathcal{B}_{2}^{\prime}(\chi)\right] \\
\varphi_{3}^{D D}= & -\mu d_{1} d_{2}\left[\mathcal{A}_{0}-3 \mathcal{B}_{2}(\chi)\right]
\end{aligned}
$$

where $\psi_{0}$ is the angle between the periastron and the node line [Gergely, Perjés, Vasúth $(1998)], \bar{\psi}=\left(\psi_{1}+\psi_{2}\right) / 2$ represents an average, $\delta=2\left(\psi_{0}-\bar{\psi}\right)$ and $\delta_{i}=\psi_{0}-\psi_{i}$ differences in the azimuthal angles. The quantty $\bar{A}$ is the magnitude of the Laplace-Runge-Lenz vector characterizing a Keplerian motion with $E$ and $\bar{L}$. Finally, $\mathcal{A}_{0}$ and $\mathcal{B}_{2}(\chi)$ denote

$$
\begin{aligned}
\mathcal{A}_{0}= & 2 \cos \lambda+3\left(\rho_{1} \sigma_{2}-\rho_{2} \sigma_{1}\right) \sin \left(\delta_{1}-\delta_{2}\right) \\
& -3\left(\rho_{1} \rho_{2}+\sigma_{1} \sigma_{2}\right) \cos \left(\delta_{1}-\delta_{2}\right), \\
\mathcal{B}_{2}(\chi)= & \left(\sigma_{1} \sigma_{2}-\rho_{1} \rho_{2}\right) \cos (2 \chi+\delta)-\left(\rho_{1} \sigma_{2}+\rho_{2} \sigma_{1}\right) \sin (2 \chi+\delta),
\end{aligned}
$$

where $\rho_{i}=\sin \alpha_{i} \cos \beta_{i}, \sigma_{i}=\sin \alpha_{i} \sin \beta_{i} \cos \kappa_{i}+\cos \alpha_{i} \sin \kappa_{i}$. Note that for the SS, QM, and DD interactions there are no contributions $\varphi_{i}$ for any $i \neq 2,3$.

It is straightforward to transform the expressions (33) into the form (13), and to identify the coefficients $f_{i 1}$ and $g_{i 1}$. From among the various emerging contributions, those entering 
in the constraint (17) are $f_{21}=\bar{A} \gamma, f_{31}=0, g_{21}=G m \mu \gamma$ and $g_{31}=-\left(\bar{L}^{2} / \mu\right) \gamma$, with $\gamma$ a complicated expression depending on the particulars of the SS, DD or QM contributions

$$
\begin{aligned}
\gamma= & \gamma_{S S}+\gamma_{Q M}+\gamma_{D D}, \\
\gamma_{Q M}= & -\frac{6 G \mu^{3} m^{3}}{2 \bar{L}^{2}} \sum_{j} p_{j} \sin ^{2} \kappa_{j} \sin 2 \delta_{j}, \\
\gamma_{S S}= & -\frac{6 G \mu^{2} S_{1} S_{2}}{c^{2} \bar{L}^{2}} \sin \kappa_{1} \sin \kappa_{2} \sin \delta, \\
\gamma_{D D}= & \frac{6 G \mu^{2} d_{1} d_{2}}{\bar{L}^{2}}\left[\left(\sigma_{1} \sigma_{2}-\rho_{1} \rho_{2}\right) \sin \left(\delta_{1}+\delta_{2}\right)\right. \\
& \left.+\left(\rho_{1} \sigma_{2}+\rho_{2} \sigma_{1}\right) \cos \left(\delta_{1}+\delta_{2}\right)\right] .
\end{aligned}
$$

It is easy to verify that the condition (17) holds in all cases, irrespective of the particular form of $\gamma$. This is why the method developed originally for constant $\varphi_{i}$-s could be employed for these cases as well

\section{The self-spin contribution to the luminosity of compact binaries}

Gravitational radiation produces dissipative effects in the orbital evolution. While the energy $E$, and the total orbital momentum $\mathbf{J}$ are conserved up to $2 \mathrm{PN}$ order accuracy (including the conservative perturbations considered in this paper and the $2 \mathrm{PN}$ relativistic perturbation), they start to evolve due to escaping gravitational radiation. This dissipative evolution can be conveniently computed with our method, presented in Section 2. The secular losses due to gravitational radiation are exactly integrals of the type (3), in which the condition of periodicity of $\omega$ is obeyed for all losses of SS, DD and QM type. As already argued in Section 3.b, the radial equation written in terms of $\bar{L}$ is also of the form (1) in all of these cases. Therefore our Theorems can be applied in computing secular radiative changes. Such secular effects were discussed in ([Gergely (2000)], [Vasúth, Keresztes, Mihály, Gergely (2003)] and [Gergely \& Keresztes (2003)]) for the SS, DD and QM contributions, respectively.

In this Section we would like to apply the method for computing the so-called selfspin contribution to the gravitational luminosity (energy loss) of compact binaries composed of black holes. For a black hole the quadrupole moment scalar is directly related to the spin: $Q_{i}=-S_{i}^{2} / m_{i}$ [Poisson (1998)]. Therefore the QM losses are effectively self-spin contributions and add to those discussed in [Gergely (2000)], [Mikóczi, Vasúth, Gergely (2005)]. Such contributions become important whenever the mass ratio of the compact 
Table 1: The coefficients $z_{i j}$ in the self-spin contribution to the luminosity.

\begin{tabular}{rrrrr}
\hline \hline$i i^{j}$ & 0 & 1 & 2 & 3 \\
\hline 0 & -539784 & -1229200 & -657120 & -47040 \\
1 & 797076 & 1807400 & 959280 & 67680 \\
2 & 266615 & 818986 & 601812 & 60600 \\
\hline
\end{tabular}

binary is at least $\eta=0.1$, which is frequently the case for colliding galactic black holes, which are the most important source types to be searched for by LISA. In such cases, as $S_{2} / S_{1} \propto \eta^{2}$, we can safely ignore $S_{2}$. Therefore the only SS interaction is the self-spin contribution.

The instantaneous energy loss can be found by taking the appropriate derivatives of the mass and current quadrupole tensors of the mass $\mu$, as described for example in [Kidder (1995)]. The self-spin contribution to the instantaneous energy loss computed in this way is a complicated expression of $r, \dot{r}$ and $\chi$. By employing the parametrization (4), we obtain $d E / d t$ as function of $\chi$ alone. The secular contribution to the energy loss arises by integration of $d E / d t$ over one radial orbit. This integration is carried out by computing the residues enclosed in the circle $\zeta=e^{i \chi}$. Due to Theorem 1, we are assured that there is only one pole, at $\zeta=0$. The summed-up self-spin contribution to the luminosity is

$$
\mathcal{L}_{Q M+S S}=-\frac{G^{2}(-2 E \mu)^{3 / 2} S^{2}}{960 c^{7} \bar{L}^{11}} \frac{m_{2}}{m_{1}}\left\{Z_{0}+\left[Z_{1}+Z_{2} \cos 2\left(\psi_{0}-\widetilde{\psi}\right)\right] \sin ^{2} \widetilde{\kappa}\right\}
$$

where

$$
Z_{i}=G^{6} m^{6} \mu^{9} \sum_{j=0}^{3} z_{i j}\left(\frac{E \bar{L}^{2}}{G^{2} m^{2} \mu^{3}}\right)^{j}, \quad i=0 . .2 .
$$

The angles $\widetilde{\kappa}$ and $\widetilde{\psi}$ are shown in Fig1. The coefficients $z_{i j}$ are enlisted in table 1. Eqs. (40)-(41) contain the totality of second order contributions in the spins to the luminosity, provided $S_{2}=0$, and as such, represent the first correction to the Lense-Thirring approach, discussed in [Gergely, Perjés, Vasúth (1998)].

\section{Concluding Remarks}

Perturbed Keplerian motions can be parametrized in many different ways. (For a review of these parametrizations see [Klioner \& Kopeikin (1994)].) The method of com- 


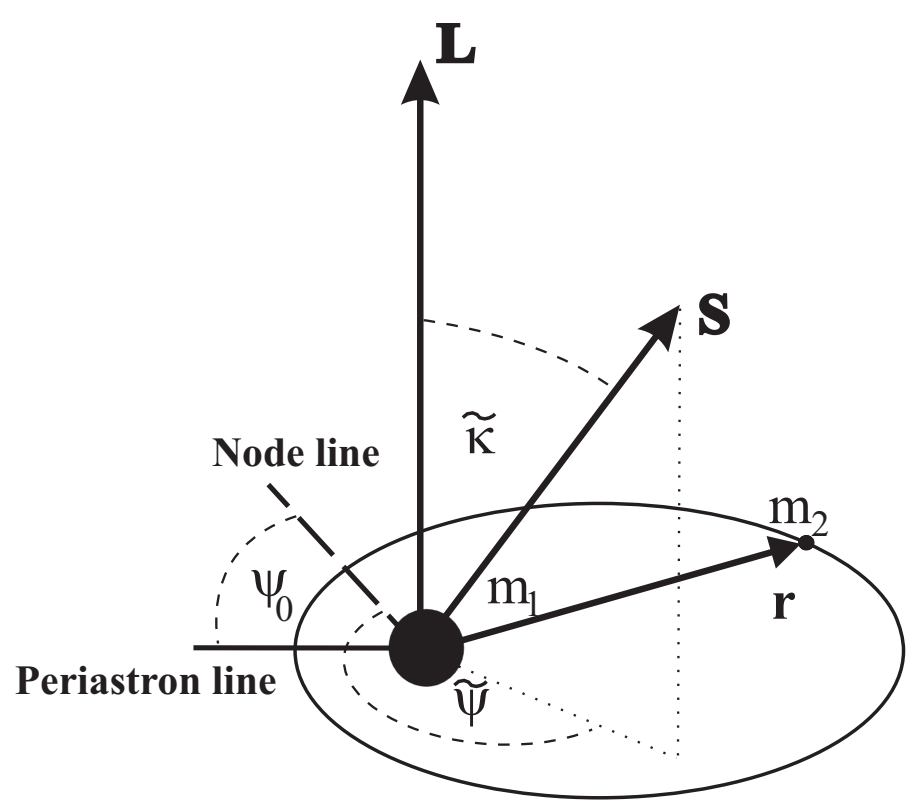

Fig. 1.- The figure represents a black hole - black hole binary system. $\mathbf{S}$ is the spin of the supermassive black hole with mass $m_{1}$, while the spin of $m_{2} \ll m_{1}$ is negligible. As there is either no $S S$ or $Q M$-contribution to the orbital angular momentum [Kidder (1995), Gergely \& Keresztes (2003)], $\mathbf{L}=\mu \mathbf{r} \times \mathbf{v}$ is the Newtonian orbital angular momentum. $\widetilde{\kappa}$ is the angle between $\mathbf{S}$ and $\mathbf{L}$. The angle between the node line and projection of the spin in the plane of the orbit is denoted as $\widetilde{\psi}$.

puting secular effects presented in [Gergely, Perjés, Vasúth (2000)] relies on novel complex parametrizations of the radial part of the motion and its advantage over previous approaches [Ryan (1996)], [Rieth \& Schäfer (1997)] is in its overwhelming simplicity. Other methods can of course be successfully applied, like the use of the Laplace second integrals for the Legendre polynomials ([Whittaker \& Watson (1996)], [Gopakumar \& Iyer (1997)]).

In the present work we have generalized both the class of integrands and the class of perturbing forces for which the simple method relying on the use of complex true and eccentric anomaly parametrizations applies.

In a generic perturbed two-body problem, it is easy to see whether a particular secular effect to be computed is of the type (3), with $\omega$ periodic, and then to check whether the periodic perturbing functions $\varphi_{i}$ obey the conditions (17). Then, depending on the power $n$, either Theorems 1 or 2 can be applied.

We have also shown explicitly, that for several physically interesting linear perturbations 
in the conservative dynamics these Theorems apply. Whether the SO, SS, QM or DD perturbations are of $1 \mathrm{PN}$ order or higher, depends on the specific system considered. For comparable mass compact binaries for example the SO contribution is of $1.5 \mathrm{PN}$ order, while the SS, QM, DD contributions are all of order $2 \mathrm{PN}$.

By contrast, when one of the masses of the compact binary dominates over the other, the SS contribution becomes negligible, however the so-called self-spin contribution of the dominant spin becomes important. Driven by this remark, as an application to the presented method, we have computed the self-spin contribution to the gravitational luninosity.

\section{Acknowledgments}

This work was supported by OTKA grants no. T046939 and TS044665. L.Á.G. wishes to thank the János Bolyai Scholarship for support.

\section{REFERENCES}

The LIGO Scientific Collaboration: Abbott, B., et al. 2004, 69, 122001

The LIGO Scientific Collaboration: Abbott, B., et al. 2005, 94, 181103

Abramovici, A., et al., Science 256, 325

Barker, B. M., O'Connell, R. F. 1979, Gen.Relativ.Gravit. D2, 1428

Bender, P., et. al. 1996 LISA: Pre-Phase A Report (MPQ 208) (Max-Planck Institut f ür Quantenoptik, Garching, Germany)

Blanchet, L. , Faye, G., Iyer, B.R., Joguet, B. 2002 Phys. Rev. D65, 061501, erratum 2005 ibid. D71, 129902

Bradaschia, C., et al. 1990, Nucl. Instrum. Methods A 289, 518

Brumberg, V. A. 1991, Essential Relativistic Celestial Mechanics. Adam Hilger, Bristol

Damour, T., Deruelle, N. 1985 Ann. Inst. Henri Poincaré A 43, 107

Gergely, L. Á., Perjés, Z. I., Vasúth, M. 1998 Phys. Rev. D57, 876; ibid. D57, 3423; ibid. D58, 124001

Gergely, L. Á., Perjés, Z. I., Vasúth, M. 2000, ApJS. 126, 79 
Gergely, L. Á. 2000, Phys. Rev. D61, 024035; ibid. 024007. Conversion to our notations: $(\bar{L}, \bar{A}) \rightarrow\left(L, A_{0}\right)$

Gergely, L. Á., Keresztes, Z. 2003, Phys. Rev. D67, 024020

Gopakumar, A., Iyer, B. R. 1997, Phys. Rev. D56, 7708

Hough, J. 1992, in Proceedings of the Sixth Marcell Grossmann Meeting, edited by H. Sato and T. Nakamura (World Scientific, Singapore), p. 192

Hulse, R. A.,Taylor, J. H. 1975, ApJ. 537, 195

Ioka, K., Taniguchi, T. 2000, ApJ. 537, 327

Keresztes, Z., Mik óczi, B., Gergely L. Á. 2005, Phys. Rev. D 72, 104022

Kidder, L., Will, C., Wiseman, A. 1993 Phys. Rev. D 47, R4183

Kidder, L. 1995 Phys. Rev. D52, 821

Klioner, S. A., Kopeikin, S. M. 1994, ApJ. 427, 951

Kuroda, K., et al. 1997 in Proceedings of International Conference on Gravitational Waves: Sources and Detectors, eds. I. Ciufolini and F. Fidecaro (World Scientific, Singapore), p. 100

Messaritaki, E. (for the LIGO Scientific Collaboration) 2005, Class.Quant.Grav. 22, S1119

Mikóczi, B., Vas úth, M., Gergely, L. Á. 2005, Phys. Rev. D71, 124043

Newton, T.D., Wigner, E.P. 1949, Rev. Mod. Phys. 21, 400

Poisson, E. 1995, Phys. Rev. D52, 5719, addendum 1997, ibid. D55, 7980

Poisson, E. 1998, Phys. Rev. D57, 5287

Pryce, M.H.L. 1948 Proc. R. Soc. A195, 62

R. Rieth, R., Schä fer, G. 1997, Class.Quant.Grav. 14, 2357

Ryan, F. D. 1996, Phys. Rev. D53, 3064

Soffel, M.N. 1988, Relativity in Astrometry, Celestial Mechanics and Geodesy, Boston: Springer-Verlag

Taylor, J. H., Fowler, L. A., McCulloch, P. M. 1979, Nature 277, 437 
Vasú th, M., Keresztes, Z., Mihály, A., Gergely, L. Á. 2003, Phys. Rev. D68, 124006

Weisberg, J.M., Taylor, J.H. 2004, APS Conference Series, 328, 25

Whittaker, E. T., Watson, G. N. A Course of Modern Analysis 1996, (4th ed. ; Cambridge: Cambridge Univ. Press) 